\title{
Absence of caspase 3 activation in neoplastic cells of nasopharyngeal carcinoma biopsies predicts rapid fatal outcome
}

\author{
Joost J Oudejans ${ }^{1}$, Ahemd Harijadi ${ }^{2}$, Saskia AGM Cillessen ${ }^{1}$, Pierre Busson ${ }^{3}$, I Bing Tan ${ }^{4}$, \\ Danny F Dukers ${ }^{1}$, Wim Vos ${ }^{1}$, Bambang Hariwiyanto ${ }^{2}$, Jaap Middeldorp ${ }^{1}$ and \\ Chris JLM Meijer ${ }^{1}$
}

\begin{abstract}
${ }^{1}$ Department of Pathology, VU Medical Center, Amsterdam, The Netherlands; ${ }^{2}$ Gadjah Mada University, School of Medicine/Sardjito Academic Hospital, Yogyakarta, Indonesia; ${ }^{3}$ UMR 8126, Institut Gustave Roussy, Villejuif, France and ${ }^{4}$ Department of Otolaryngology of the Antoni van Leeuwenhoekhuis/The Dutch Cancer Institute, Amsterdam, The Netherlands
\end{abstract}

\begin{abstract}
Poor prognosis in nasopharyngeal carcinoma patients may result from resistance to the apoptosis-inducing effect of radio- and/or chemotherapy. Apoptosis depends on proper activation of caspase 3 , resulting in cleavage of key proteins like PARP-1. To investigate whether disruption of the apoptosis pathway results in therapy-resistant tumour cells, we investigated whether absence of caspase 3 activation in tumour biopsies of nasopharyngeal carcinoma patients is related to poor clinical outcome. Moreover, we investigated whether absence of caspase 3 activation is related to loss of procaspase 3 expression or expression of the apoptosis regulators p53, bcl-2 and XIAP. We studied 36 Indonesian nasopharyngeal carcinoma patients without evidence of distant metastases who were treated with curative intent by radiotherapy only. Activation of caspase 3 and expression of the different markers were determined using specific antibodies. Levels of caspase 3 activation were determined by quantifying positively staining tumour cells. Nasopharyngeal carcinoma-derived C15 and C17 tumour cells were used as control. Absence of caspase 3 activation was strongly related to a poor clinical response to radiotherapy and to a higher $\mathrm{T}$ and $\mathrm{N}$ stage, resulting in a particularly poor clinical outcome with regard to progression-free $(P<0.0001)$ and overall survival time $(P<0.0001)$. Absence of caspase 3 activation was significantly correlated to loss of expression of procaspase $3(P=0.04)$. In nasopharyngeal carcinoma patients treated with curative intent, absence of active caspase 3-positive neoplastic cells predicts rapid fatal outcome, and is associated with poor response to radiotherapy and high $\mathbf{T}$ and $\mathbf{N}$ stage at time of presentation. Modern Pathology (2005) 18, 877-885. doi:10.1038/modpathol.3800398; Published online 1 April 2005
\end{abstract}

Keywords: prognosis; apoptosis; caspase; XIAP; bcl-2; nasopharyngeal carcinoma

Nasopharyngeal carcinoma is relatively rare in Caucasians, but occurs at high frequency in Southern China and South East Asia. In South East Asia nasopharyngeal carcinoma is a major cause of cancer deaths. ${ }^{1}$ Nasopharyngeal carcinoma is a highly radiosensitive tumour, and by modern imaging and radiation techniques, local control rates of greater than $80 \%$ are obtained, even in patients with high tumour volumes. ${ }^{2}$ Still distant failures remain the major problem in patients with locoregional bulky disease, which is the most common form at

Correspondence: Dr JJ Oudejans, MD, PhD, Department of Pathology, VU Medical Centre, De Boelelaan 1117, Amsterdam 1081 HV, The Netherlands.

E-mail: jj.oudejans@azvu.nl

Received 6 December 2004; revised and accepted 25 January 2005; published online 1 April 2005 presentation. $^{3}$ At present, 5-year survival rates of more than $50 \%$ are obtained, but prognosis depends strongly on stage. ${ }^{3}$

Although nasopharyngeal carcinoma is a radiosensitive tumour, a large proportion of tumours relapse with increasing resistance to radio- and or chemotherapy. In vitro data indicate that the cell death-inducing effect of radio- and chemotherapy depends on activation of the apoptosis cascade also in nasopharyngeal carcinoma cells. ${ }^{4-9}$ Thus, it is conceivable that the tumours that fail to respond to therapy have a defective apoptosis cascade. Apoptosis is a form of cellular suicide characterised by distinct morphological recognisable phases. It can be triggered by a variety of stimuli, including cytotoxic T-lymphocyte-mediated killing through either the CD95 or granzyme B/perforin-mediated pathway, and also by ionising radiation and many 
cytostatic drugs. ${ }^{10}$ Upon induction of apoptosis, a cascade of proteases called caspases (cystein-containing aspartic acid-specific proteases) is activated. ${ }^{10}$ In vitro studies have elucidated two major apoptosis pathways: (1) a stress-induced pathway via activation of caspase $9^{11-13}$ and (2) a death receptor-mediated pathway via activation of caspase 8. ${ }^{14,15}$ Both pathways induce apoptosis via activation of caspase 3 and possibly other effector caspases (6 and 7), which execute cell death through degradation of vital proteins. ${ }^{10}$ Granzyme B can directly activate caspase 3 or indirectly via activation of caspase 9 by truncating Bid. ${ }^{16-20}$

Thus, all pathways depend upon activation of effector caspases, in particular caspase 3, for the final execution of apoptosis. Therefore, it might be expected that high levels of active caspase 3 reflect proper functioning of one or both identified apoptosis pathways, resulting in relatively chemotherapy-sensitive neoplastic cells and a favourable response to chemotherapy.

We tested this hypothesis by quantifying numbers of active caspase 3-positive neoplastic cells on paraffin-embedded tissue sections of diagnostic biopsies of 36 nasopharyngeal carcinoma patients taken prior to the start of radiotherapy and comparing percentages of active caspase 3-positive neoplastic cells to clinical outcome. In addition, we investigated whether low numbers of active caspase 3 -positive cells were correlated with high expression levels of the apoptosis-inhibiting proteins bcl-2 and XIAP or with loss of procaspase 3 expression. Furthermore, functional analysis of apoptosis sensitivity was tested in nasopharyngeal carcinomaderived C15 and C17 tumour cells to investigate whether aberrant expression of bcl-2, XIAP and/or procaspase 3 correlates with resistance to chemotherapy and/or irradiation-induced apoptosis.

\section{Materials and methods}

\section{Patient Selection}

In all, 36 patients were selected from 202 patients, diagnosed between 1995 and 1996 at the Department of Pathology, Gadjah Mada University, School of Medicine/Sardjito Academic Hospital, Yogyakarta, Indonesia. For selection of patients, see Figure 1. Only patients without evidence for distant metastases at the time of diagnosis were included (as determined by clinical and ear/nose/throat examination and by chest X-ray). In addition, only patients were entered who were treated with curative intent with radiotherapy only and with adequate follow-up including clinical and ear/nose/ throat examination and nasopharynx cytology every 3 months. A computed tomography scan was not routinely performed. Of the 202 patients, 87 patients presented with advanced stage of disease and distant metastasis, and 55 patients did not have adequate follow-up and complete radiotherapy

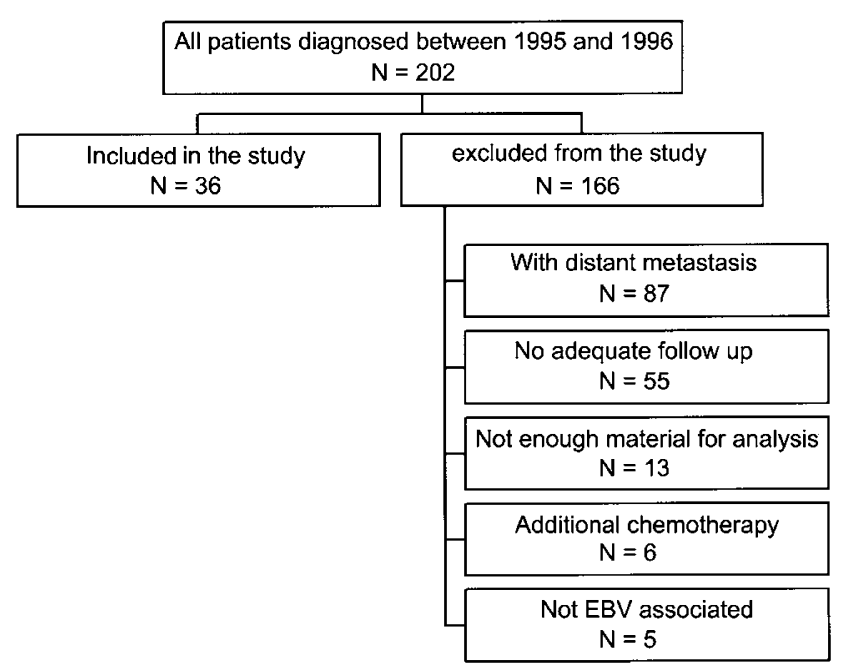

Figure 1 Flow-chart of selection of nasopharyngeal carcinoma patients included in this study.

according to the protocol, including all patients with T4 carcinoma. A total of 13 patients did not have pathologic tissue available for immunohistochemical staining. Six patients were excluded because they received additional treatment with Cisplatin. Epstein-Barr virus-negative carcinomas $(N=5)$ were excluded by EBER in situ hybridisation using PNA probes (DakoCytomation, Glostrup, Denmark) to exclude possible cases of sinonasal undifferentiated carcinoma. The medical records of the remaining 36 patients were reviewed for all relevant clinical data including, if necessary, correspondence with patients/families or home visits. Tumour staging was performed according to the International Union Against Cancer (UICC) system. ${ }^{21}$ All patients received a radiation of $60-70 \mathrm{G}$, divided in 30-35 fractions: five fractions a week using Cobalt 60 radiotherapy equipment. Only nonkeratinising carcinomas were included in our study. Cases were classified according to the 1991 WHO Histological Classification $^{22}$ into differentiated nonkeratinising carcinoma, $N=7$, and undifferentiated carcinoma, $N=29$. The histological discrimination between these two subtypes did not have any prognostic value. The institutional review board of the VU University Medical Centre approved the study. Informed consent was provided according to the Declaration of Helsinki.

\section{Detection of Pro- and Active Caspase 3, Cleaved PARP-1/p89, Bcl-2 and XIAP}

In all, $4 \mu \mathrm{m}$ thick sections from the paraffinembedded biopsies were stained using a standard three-step streptavidin-biotin-complex method, with $3,3^{\prime}$-diaminobenzidine. The following monoclonal antibodies were used: monoclonal rabbitantiactive caspase 3 (Pharmingen, San Diego, $\mathrm{USA}^{23}$ ), polyclonal Rabbit anti-p89 (Promega Cor- 
poration, MD, USA) detecting the cleaved fragment form of PARP-1, ${ }^{23-25}$ monoclonal antiprocaspase 3 (clone 19, Coulter, Hialeah, USA), Polyclonal antiprocaspase 3 (CPP32, DakoCytomation, Glostrup, Denmark), anti-Bcl-2 (DakoCytomation, Glostrup, Denmark), anti-XIAP (MBL, Nagoya, Japan) and p53 (DO-7, DakoCytomation, Glostrup, Denmark). Since the DO-7 antibody detects both wild-type and mutated p53, expression of its downstream effector molecule p21 was determined as an indirect reflection of its functionality using anti-p21 (Oncogene Science Inc., Cambridge, MA, USA). All antibodies required antigen retrieval by microwave irradiation for $10 \mathrm{~min}$ in a citrate buffer $(10 \mathrm{mM} / \mathrm{l}, \mathrm{pH} 6.0$ at $700 \mathrm{~W}$ ), after which sections were incubated for $1 \mathrm{~h}$ at room temperature (most antibodies) or incubated overnight (Bcl-2). For all antibodies, staining intensity was enhanced using the catalysed reporter deposition (CARD) method (DakoCytomation, Glostrup, Denmark).

\section{Phenotypical Analysis of Active Caspase 3-Positive Cells}

In four cases, double stainings were performed for active caspase 3 and the keratin-specific antibody AE1/3. Primary antibodies were incubated simultaneously, followed first by detection of active caspase 3 with a biotinylated polyclonal donkey-anti-rabbit antibody and visualised with $3,3^{\prime}$-diaminobenzidine and second by detection of AE1/3 Dako) with an alkaline phosphatase-conjugated rabbit-anti-mouse antibody, visualised with new fuchsin/naphtol alkaline phosphatase biphosphate. Negative controls included simultaneously processed sections with omission of the active caspase 3- and AE1/3-specific antibodies, respectively.

\section{Quantification of Neoplastic Cells Expressing Apoptosis-Related Proteins}

Percentages of active caspase 3-positive cells were quantified using a microscopic grid and were expressed as percentages of all tumour cells present. To avoid counting of active caspase 3-positive reactive lymphocytes, only unequivocally neoplastic cells with large nuclei (more than three times the size of a lymphocyte) were counted. Moreover, only cells with nuclear staining were counted to avoid counting macrophages with phagocytosed apoptotic debris. Expression of procaspase 3, Bcl-2- and XIAP was scored as either positive or negative.

\section{Analysis of Clinical Data}

For each patient, the following characteristics were noted from the medical records: age, sex, clinical TNM stage, therapy, response and occurrence of relapses and cause of death. Complete follow-up data of all patients until time of death or until 52 months after initial diagnosis, were available, when patients were discharged from follow-up. Survival time was measured from time of initial diagnosis until death or end of follow-up. All deceased patients had evidence of tumour recurrence at time of death and were considered to have died as a result of the tumour. Progression-free survival time was measured from time of initial diagnosis until time of disease relapse.

\section{Statistical Methods}

Survival curves were constructed with the KaplanMeier method. Differences between the curves were analysed using the Log-rank test. Multivariate analysis was performed using the Cox-proportional hazards model (enter and remove limits 0.1). ${ }^{26}$ Comparisons of means were performed using the Mann-Whitney $U$ test. Categorical variables were analysed by Pearson $\chi^{2}$ test or by Fisher's exact test, when appropriate. All $P$-values are based on twotailed statistical analysis. $P$-values below 0.05 were considered as significant. All analyses were performed using the SPSS statistical software (version 11.5 SPSS Inc., Chicago, USA).

\section{Determination of the Functional Integrity of the Caspase 9-Mediated Apoptosis Pathway}

To validate the immunohistochemical results obtained on nasopharyngeal carcinoma biopsies, we tested the sensitivity of nasopharyngeal carcinomaderived cell lines C15 and C17 cells for stressinduced apoptosis. For this, C15 and C17 cells were incubated with 25-200 $\mu \mathrm{M}$ etoposide (VP16, Sigma, USA) for 0, 1, 4, 24 and $48 \mathrm{~h}$. Etoposide was used, since it is a strong inducer of apoptosis in many different model systems, primarily via activation of the stress-induced, caspase 9-mediated pathway. ${ }^{27}$ It is highly suitable for in vitro induction of apoptosis since short incubation times suffice to induce apoptosis, and it lacks autofluorescence and thus does not interfere with fluorescent analysis.

Caspase 3-like effector caspase activity was determined using a fluorimetric homogeneous caspase assay (Roche, Mannheimm, Germany), according to the manufacturer's instructions. Cells were lysed and incubated with DEVD-rhodamine 110 substrate for $1 \mathrm{~h}$ at $37^{\circ} \mathrm{C}$. Subsequently, the amount of free rhodamine was determined fluorimetrically. The developed fluorochrome was proportional to the concentration of activated caspases and could be quantified by a calibration curve of diluted free rhodamine. Positive controls included peripheral blood mononuclear cells and B-cell lines with previously demonstrated sensitivity to etoposideinduced apoptosis.

In addition, to test sensitivity of nasopharyngeal carcinoma cells for irradiation-induced apoptosis, 
C15 tumours were propagated in nude mice ${ }^{28}$ and irradiated in duplicate with a dosage of $0,2.5,5.0$ and $10.0 \mathrm{~Gy}$. The irradiation was delivered using an RT 250 Philips $(0.69 \mathrm{~Gy} / \mathrm{min})$ under a tension of $200 \mathrm{kV}, 200 \mathrm{~mA}$, with a $0.2 \mathrm{~mm}$ copper filter. At 24 and $48 \mathrm{~h}$ postradiation, tumours were removed and snap-frozen in liquid nitrogen. Frozen sections were cut and processed for active caspase 3 detection or analysed for PARP-1/p89 cleavage as recently described. ${ }^{23}$

\section{Results}

\section{Clinical Characteristics}

Patient characteristics are summarised according to the percentage of active caspase 3-positive neoplastic cells (Table 1). In comparison to studies describ-

Table 1 Patient characteristics according to the presence of active caspase 3 -positive neoplastic cells

\begin{tabular}{|c|c|c|c|}
\hline & \multicolumn{2}{|c|}{ Active caspase 3-positive neoplastic cells } & \multirow[t]{2}{*}{ P-value } \\
\hline & Negative $(\mathrm{N}=10)$ & Positive $(\mathrm{N}=26)$ & \\
\hline Age (mean) & 53 & 43 & 0.04 \\
\hline $\operatorname{Sex}(M / F)$ & $7 / 3$ & $19 / 7$ & n.s. \\
\hline \multicolumn{4}{|l|}{$\mathrm{T}$} \\
\hline 1 & 0 & 7 & 0.01 \\
\hline 2 & 1 & 10 & \\
\hline 3 & 9 & 9 & \\
\hline \multicolumn{4}{|l|}{$\mathrm{N}$} \\
\hline 0 & 0 & 4 & 0.008 \\
\hline 1 & 0 & 11 & \\
\hline 2 & 7 & 10 & \\
\hline 3 & 3 & 1 & \\
\hline \multicolumn{4}{|c|}{ Complete remission } \\
\hline Yes & 2 & 25 & $<0.0001$ \\
\hline No & 8 & 1 & \\
\hline \multicolumn{4}{|l|}{ Relapse $^{\mathrm{a}}$} \\
\hline Yes & 2 & 18 & n.s. \\
\hline No & 0 & 7 & \\
\hline \multicolumn{4}{|l|}{ Death } \\
\hline Yes & 10 & 19 & n.s. (0.08) \\
\hline No & 0 & 7 & \\
\hline
\end{tabular}

${ }^{\mathrm{a} O n l y}$ nasopharyngeal carcinoma biopsies from patients who achieved complete remission were analysed.

n.s. $=$ not significant . ing clinical outcome in European, ${ }^{29}$ Chinese and Hong Kong patients, ${ }^{30,31}$ overall survival time of our group of patients was very poor with a median survival time of only 26 months. Although, in general, no well-documented data are available for Indonesian patients, the relatively poor outcome in our group of patients can be explained, in part, by the fact that no intracavitary afterloading treatment was performed for local persistence and that none of the patients in our study group received salvage treatment in case of tumour relapse.

\section{Caspase 3 is Activated in Low Percentages of Neoplastic Cells of Nasopharyngeal Carcinoma Biopsies}

In all of the 36 tested cases, active caspase 3-positive infiltrating lymphocytes were detected, serving as an internal positive control. In 26 cases also active caspase 3-positive neoplastic cells were observed, albeit in a very limited number of cells, with a maximum of $3 \%$ of all tumour cells (see Figure 2a). In some cases also granular cytoplasmic staining in macrophages was observed, probably representing phagocytosed apoptotic debris.

The percentage of active caspase 3-positive tumour cells ranged from $<1$ to $3 \%$, displaying a mainly nuclear staining pattern usually in cells with morphological features of apoptosis, that is, with chromatin condensation and decrease or loss of cytoplasm. Furthermore, similar to our previous study in lymphomas, ${ }^{23}$ numbers of active caspase 3 -positive tumour cells were similar to numbers of PARP-1/p89-positive tumour cells, suggesting proper functioning of active caspase 3 (Figure 2a).

Absence of caspase 3 activation was related to expression of XIAP, loss of procaspase 3 expression and expression of bcl-2. Expression of XIAP and bcl2 was detected in 14 and 26 of 36 tested cases, respectively, as diffuse cytoplasmic staining in the large majority of tumour cells (see Figure 2). Expression of XIAP tended to be expressed more frequently in active caspase 3-negative cases (see Table 2). In contrast, bcl-2 was significantly more frequently expressed in active caspase 3-positive cases. A significant correlation between absence of procaspase 3 expression and absence of caspase 3 activation was found; in four of 10 active caspase 3negative cases, no procaspase 3 was found, whereas procaspase could easily be detected in 24 of 26

Figure 2 Immunohistochemical detection of apoptosis regulating/executing proteins in biopsy specimens: (a) Active caspase 3 staining displaying a mainly nuclear staining pattern usually in large neoplastic cells with morphological features of apoptosis, that is, with chromatin condensation and decrease or loss of cytoplasm. Inset shows staining for the cleaved PARP-1/p89 fragment also in cells with morphological features of apoptosis. (b) Pro-caspase 3-positive staining showing cytoplasmic staining using the polyclonal antibody CPP32. (c) Pro-caspase 3-positive staining using the monoclonal antibody on the same biopsy as shown in (b). (d) Nasopharyngeal carcinoma biopsy showing loss of procaspase 3 expression using both antibodies, with clear expression in tumour infiltrating small lymphocytes (shown is the staining using the polyclonal antibody CPP32). (e) XIAP expression demonstrating the characteristic granular cytoplasmic staining in positive cases and (f) absence of staining in a negative nasopharyngeal carcinoma, infiltrating reactive lymphocytes serve as internal positive control (arrowheads). (g) Bcl-2 expression in a strong positive nasopharyngeal carcinoma and (h) a negative case with positive tumour infiltrating lymphocytes, again infiltrating reactive lymphocytes served as internal positive controls. 

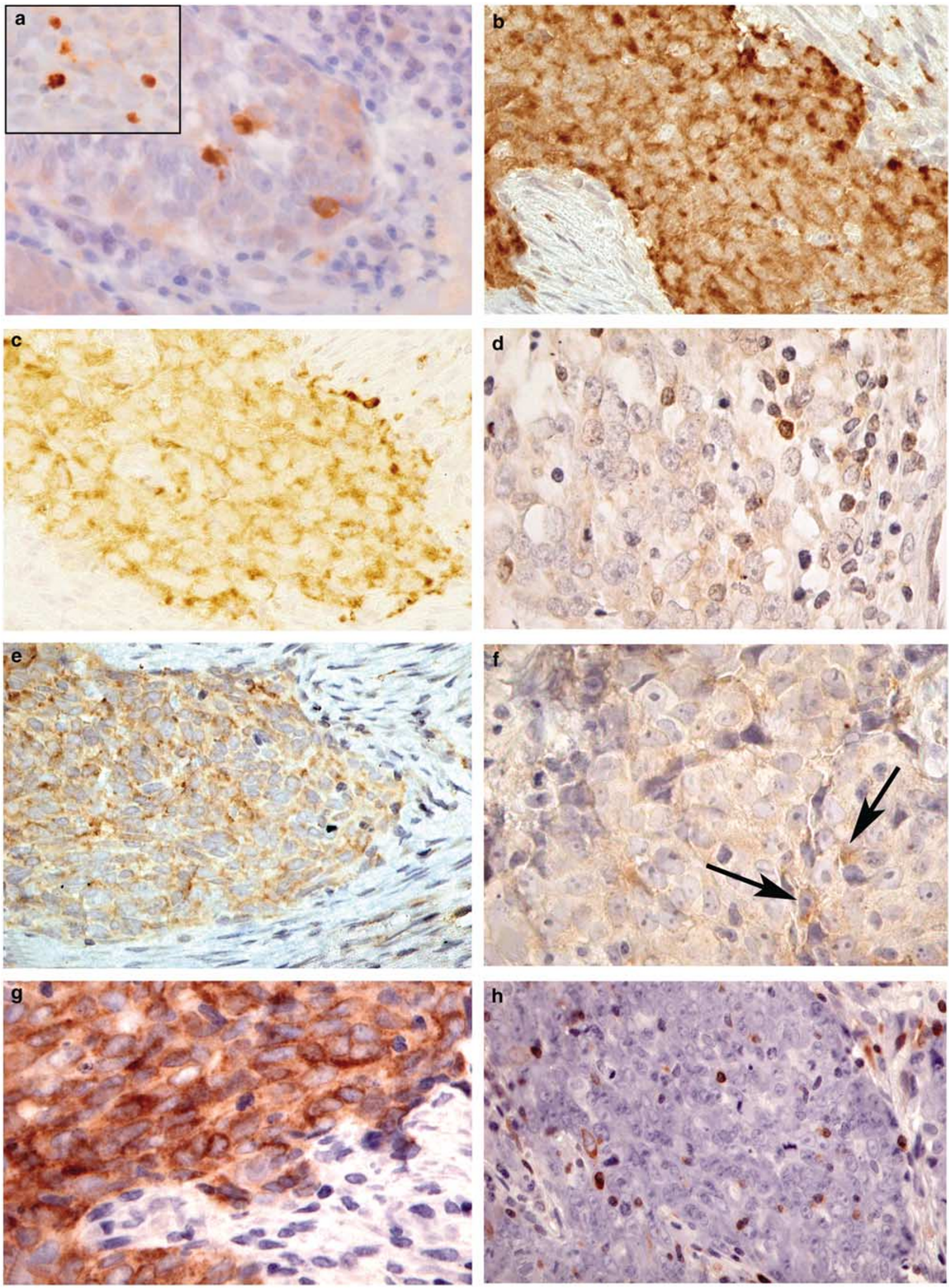
Table 2 Tumour characteristics according to the presence of active caspase 3-positive neoplastic cells

\begin{tabular}{|c|c|c|c|}
\hline & \multicolumn{2}{|c|}{$\begin{array}{c}\text { Active caspase } 3 \text {-positive } \\
\text { neoplastic cells }\end{array}$} & \multirow[t]{2}{*}{$\mathrm{P}$-value } \\
\hline & \multicolumn{2}{|c|}{ Negative $(\mathrm{N}=10)$ Positive $(\mathrm{N}=26)$} & \\
\hline \multicolumn{4}{|l|}{ Bcl-2 } \\
\hline Negative & 6 & 4 & 0.01 \\
\hline Positive & 4 & 22 & \\
\hline \multicolumn{4}{|l|}{ XIAP } \\
\hline Negative & 4 & 18 & n.s. (0.1) \\
\hline Positive & 6 & 8 & \\
\hline \multicolumn{4}{|l|}{ Procaspase 3} \\
\hline Negative & 4 & 2 & 0.04 \\
\hline Positive & 6 & 24 & \\
\hline \multicolumn{4}{|l|}{ P53 } \\
\hline Negative & 2 & 2 & n.s. \\
\hline Positive & 8 & 24 & \\
\hline \multicolumn{4}{|l|}{$P 21$} \\
\hline Negative & 4 & 6 & n.s. \\
\hline Positive & 6 & 20 & \\
\hline $\begin{array}{l}\text { Mean \% granzyme } \\
\text { B+tumour infiltrating } \\
\text { lymphocytes (s.d.) }\end{array}$ & $53(18)$ & $21(14)$ & $<0.001$ \\
\hline
\end{tabular}

s.d. = standard deviation

active caspase 3-positive cases (see Table 2). No significant relation between caspase 3 activation and either expression of p53 or p21 was observed.

\section{Absence of Caspase 3 Activation in Neoplastic Nasopharyngeal Carcinoma Cells is Related to Poor Response to Therapy, Early Relapse and Rapid Fatal Clinical Outcome}

Using Cox regression analysis, the influence on overall survival time of percentage of active caspase 3-positive tumour cells was estimated. Prognosis was found to be more favourable in cases with higher percentages of active caspase 3-positive tumour cells (entered as continuous variable, $P=0.001$ ). When patients were subdivided into a group with or without active caspase 3-positive tumour cells, absence of active caspase 3-positive tumour cells identified a group of patients with a particular unfavourable prognosis (Log rank test; $P<0.0001$, see Figure 3). Absence of caspase 3 activation was also strongly related to higher age, higher $\mathrm{T}$ and $\mathrm{N}$ stage and failure to achieve complete remission (see Table 1).

\section{Disruption of Apoptosis Pathways in Nasopharyngeal Carcinoma-Derived Tumour Cell Lines}

To correlate our immunohistochemical results with functional sensitivity to apoptosis, we investigated expression levels of bcl-2, XIAP and procaspase 3 in

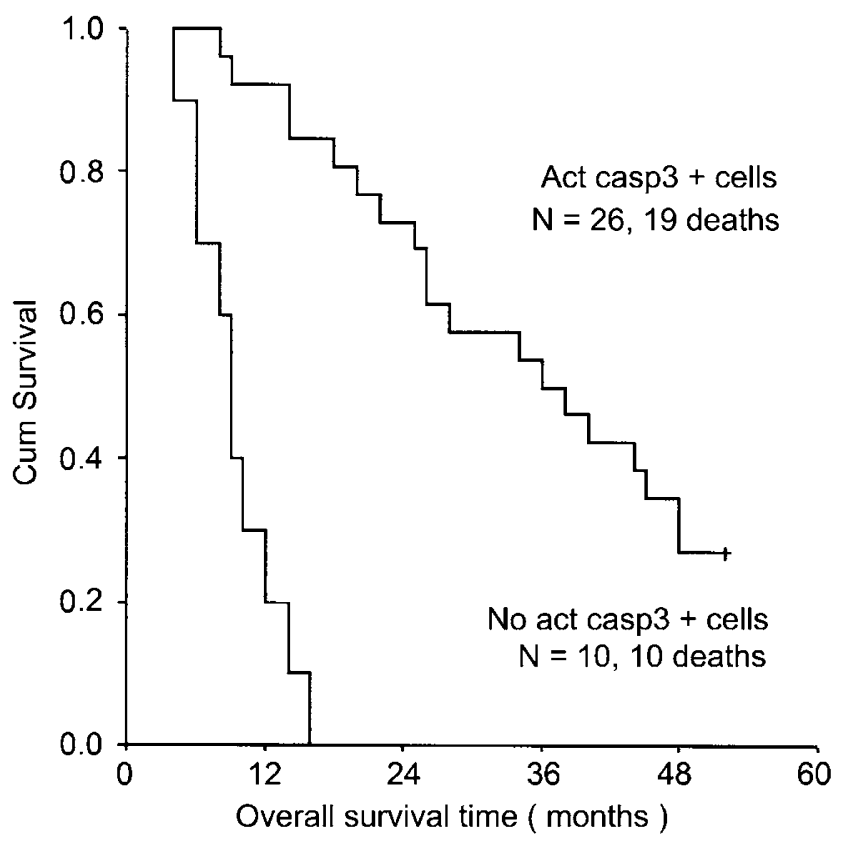

Figure 3 Overall survival analysis of nasopharyngeal carcinoma patients according to the presence or absence of active caspase 3positive tumour cells. The observed difference was significant (Log-Rank test, $P<0.0001$ ).

nasopharyngeal carcinoma-derived cell lines and tested the sensitivity of these cell lines to etoposide and irradiation therapy-induced apoptosis and caspase 3 activation. Both tested nasopharyngeal carcinoma-derived cell lines showed clear expression of both bcl-2 and XIAP. One of these cell lines (C15) also demonstrated nearly complete loss of procaspase 3 expression (see Figure 4). Using etoposide we could not induce caspase 3 activation in both tested nasopharyngeal carcinoma cell lines even when very high concentrations and/or long incubation periods were used. Cytospin preparations confirmed complete absence of cells with apoptotic morphology following induction with etoposide (data not shown).

Similarly, tissue sections prepared from C15 tumours grown in nude mice failed to show any active caspase-3 expression or caspase-3-mediated PARP-1 cleavage at 24 or $48 \mathrm{~h}$ following 'in vivo' irradiation with 2.5-10 Gy (data not shown).

\section{Absence of Caspase 3 Activation is Related to the Presence of High Percentages of Activated Cytotoxic T Lymphocytes}

We have previously demonstrated that high percentages of granzyme B-positive tumour infiltrating lymphocytes are also highly predictive of a rapid fatal outcome in this same group of nasopharyngeal carcinoma patients. ${ }^{32}$ As expected, a strong inverse relationship between both variables was found. In 

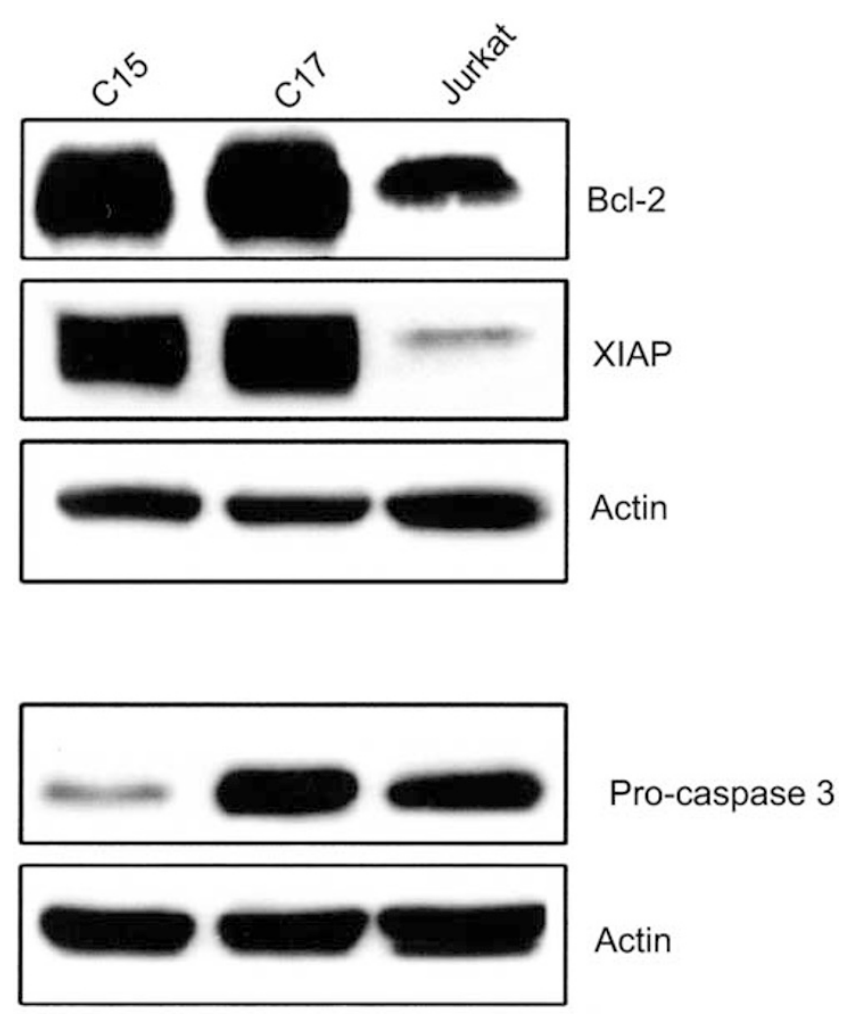

Figure 4 Detection of apoptosis-regulating proteins in two nasopharyngeal carcinoma-derived cell lines by Western blot analysis: representative results of three independent experiments are shown. The Jurkat T-cell line was used as positive control. Actin expression levels demonstrate equal protein loading concentrations for the three tested cell lines. Strong expression of bcl-2 and XIAP was detected in C15 and C17. One of both nasopharyngeal carcinoma lines (C15) demonstrated very low levels of procaspase 3 expression as compared to C17 and Jurkat.

cases without active caspase 3-positive tumour cells, relatively very high levels of granzyme Bpositive tumour infiltrating lymphocytes were detected as compared to active caspase 3-positive cases (see Table 2).

\section{Multivariate Analysis of Overall Survival Time}

The Cox-proportional hazards model was used for multivariate analysis. Variables included were the presence or absence of active caspase 3-positive cells, age (entered as continuous variable) and $\mathrm{T}$ and $\mathrm{N}$ stage. The predictive value of active caspase 3-positive tumour cells was partly related to the $\mathrm{T}$ stage but retained its prognostic power $(P=0.05)$. If also included, the percentage of granzyme B-positive tumour infiltrating lymphocytes was the strongest prognostic marker.

\section{Discussion}

We have shown that the complete absence of caspase 3 activation in pretreatment biopsies of nasopharyngeal carcinoma patients is strongly associated with a very poor clinical outcome. Active caspase 3-positive cells were usually morphologically recognisable as being (pre-) apoptotic, and numbers of active caspase 3-positive tumour cells were similar to numbers of cleaved PARP-1/p89positive tumour cells, suggesting proper functioning of active caspase 3 and execution of apoptosis by activated caspase 3 .

The strong relation between absence of active caspase 3-positive tumour cells and poor prognosis supports our notion that an intact apoptosis pathway is necessary for a favourable response to radio- and/or chemotherapy. ${ }^{23}$ This is particularly illustrated by the fact that the large majority of patients with absence of active caspase 3-positive tumour cells failed to achieve complete remission. Moreover, a significant correlation was observed with higher $\mathrm{T}$ and $\mathrm{N}$ stage consistent with a previous study. ${ }^{33}$ Assuming that in these cases apoptosis is disrupted, apoptosis-resistant tumour cells may have better opportunities for survival in lymph nodes than cells with a relatively intact apoptosis cascade. A similar mechanism has been postulated for malignant melanomas. ${ }^{34}$

We have previously demonstrated that high numbers of granzyme B-positive tumour infiltrating lymphocytes strongly predict rapid fatal outcome in this same group of patients (see Oudejans et $a l^{32}$ ). Apparently, these tumour infiltrating lymphocytes fail to effectively kill the tumour cells. Several immune escape mechanisms may be involved in this immune failure including loss of MHC class I expression and expression of the granzyme B-inhibitor PI-9 by the tumour cells. ${ }^{32}$ Alternatively, disruption of the apoptosis pathway would also lead to complete resistance to cytotoxic T-lymphocyte-induced cell death. The strong relationship between high numbers of granzyme B-positive tumour infiltrating lymphocytes and absence of caspase 3-positive tumour cells suggests that this putative immune escape mechanism may be important in a significant part of nasopharyngeal carcinoma cases. Thus, in nasopharyngeal carcinoma cases with a strong cytotoxic T-lymphocytemediated immune response, a selection might occur for tumour cells that manage to escape from activated cytotoxic $\mathrm{T}$ lymphocytes. If immune escape of tumour cells is (also) achieved by disruption of the apoptosis pathway this will result in crossresistance to radiotherapy (and chemotherapy) induced apoptosis and thus in a poor clinical outcome, as has been demonstrated previously in in vitro models. ${ }^{35,36}$

In cases with a poor response to chemotherapy, the low levels of active caspase 3 would be expected to reflect disruption of the caspase 9 pathway since this is the pathway primarily involved in radiotherapy-induced apoptosis. ${ }^{37}$ This is consistent with our finding that nasopharyngeal carcinoma-derived cell lines did not show any caspase 3 activation and 
apoptosis when induced by etoposide and irradiation, which is also consistent with a recent report demonstrating that also doxorubicin, taxol and cis-platinum failed to induce apoptosis in nasopharyngeal carcinoma-derived cell lines. ${ }^{28}$

From the different tested apoptosis regulating proteins, loss of procaspase 3 expression was significantly correlated with absence of active caspase 3 and also XIAP tended to be expressed more frequently in active caspase 3-negative cases. Bcl-2 expression was also frequently expressed but in particular in active caspase 3-positive cases. Expression of p53 was frequently detected in both active caspase 3-positive and -negative cases. The mutational status of p53 cannot be assessed using the DO-7 antibody, but the concomitant detection of p21 in the majority of p53-positive cases suggests that p53 is most likely present in its wild-type form, consistent with previous reports. ${ }^{38,39}$ Together these data suggest that in nasopharyngeal carcinoma the caspase 9 pathway is activated via upregulation of p53 but is disrupted downstream from caspase 9 activation by expression of XIAP or by loss of procaspase 3 expression. Still many other apoptosis-regulating proteins might also be involved in disruption of the apoptosis pathway including other members of the IAP ${ }^{40}$ and bcl-2 protein family (including the Epstein-Barr virus encoded bcl-2 homologue BHRF-1. ${ }^{41-43}$

If specific disruption of the caspase 9-mediated pathway is indeed a major factor in radiotherapy resistance, nasopharyngeal carcinoma patients might benefit from specifically triggering the death receptor-induced caspase 8-mediated pathway by the TNF-related apoptosis-inducing ligand (TRAIL/ Apo2L). This approach has been shown to be feasible in different model systems, ${ }^{44,45}$ and may be feasible also in nasopharyngeal carcinoma since it was recently reported that the caspase 8 pathway is fundamentally intact in nasopharyngeal carcinoma cells. ${ }^{46}$

We conclude that, in nasopharyngeal carcinoma patients treated with curative intent, absence of active caspase 3-positive neoplastic cells predicts rapid fatal outcome, and is associated with poor response to radiotherapy and high $\mathrm{T}$ and $\mathrm{N}$ stage at time of presentation. These data support the notion that in nasopharyngeal carcinoma the apoptosis cascade is involved in the tumour cell-killing effect of radio- and chemotherapy and in the ability of tumour cells to survive in lymph nodes. Our findings may be of relevance for the design of novel therapeutic approaches in nasopharyngeal carcinoma.

\section{Acknowledgements}

This work was supported by the Pieter van Foreest Foundation and by the Dutch Cancer Foundation.

\section{References}

1 Spano JP, Busson P, Atlan D, et al. Nasopharyngeal carcinomas: an update. Eur J Cancer 2003;39: 2121-2135.

2 Teo PM, Chan AT. Treatment strategy and clinical experience. Semin Cancer Biol 2002;12:497-504.

3 Fandi A, Cvitkovic E. Biology and treatment of nasopharyngeal cancer. Curr Opin Oncol 1995;7:255-263.

4 Friesen C, Herr I, Krammer PH, et al. Involvement of the CD95 (APO-1/FAS) receptor/ligand system in druginduced apoptosis in leukemia cells. Nature Med 1996;2:574-577.

5 Los M, Herr I, Friesen C, et al. Cross-resistance of CD95- and drug-induced apoptosis as a consequence of deficient activation of caspases (ICE/Ced-3 proteases). Blood 1997;90:3118-3129.

6 Friesen C, Fulda S, Debatin KM. Induction of CD95 ligand and apoptosis by doxorubicin is modulated by the redox state in chemosensitive- and drug-resistant tumor cells. Cell Death Differ 1999;6:471-480.

7 Belka C, Marini P, Lepple-Wienhues A, et al. The tyrosine kinase lck is required for CD95-independent caspase-8 activation and apoptosis in response to ionizing radiation. Oncogene 1999;18:4983-4992.

8 Wesselborg S, Engels IH, Rossmann E, et al. Anticancer drugs induce caspase-8/FLICE activation and apoptosis in the absence of CD95 receptor/ligand interaction. Blood 1999;93:3053-3063.

9 Tan G, Heqing L, Jiangbo C, et al. Apoptosis induced by low-dose paclitaxel is associated with p53 upregulation in nasopharyngeal carcinoma cells. Int J Cancer 2002;97:168-172.

10 Rathmell JC, Thompson CB. The central effectors of cell death in the immune system. Annu Rev Immunol 1999;17:781-828.

11 Jurgensmeier JM, Xie Z, Deveraux Q, et al. Bax directly induces release of cytochrome c from isolated mitochondria. Proc Natl Acad Sci USA 1998;95:4997-5002.

12 Slee EA, Harte MT, Kluck RM, et al. Ordering the cytochrome c-initiated caspase cascade: hierarchical activation of caspases-2, $-3,-6,-7,-8$, and -10 in a caspase-9-dependent manner. J Cell Biol 1999;144: 281-292.

$13 \mathrm{Li}$ PF, Dietz R, von Harsdorf R. P53 regulates mitochondrial membrane potential through reactive oxygen species and induces cytochrome c-independent apoptosis blocked by Bcl-2. EMBO J 1999;18: 6027-6036.

14 Stennicke HR, Jurgensmeier JM, Shin H, et al. Procaspase-3 is a major physiologic target of caspase-8. J Biol Chem 1998;273:27084-27090.

15 Darmon AJ, Nicholson DW, Bleackley RC. Activation of the apoptotic protease CPP32 by cytotoxic T-cellderived granzyme B. Nature 1995;377:446-448.

16 Darmon AJ, Ley TJ, Nicholson DW, et al. Cleavage of CPP32 by granzyme B represents a critical role for granzyme $\mathrm{B}$ in the induction of target cell DNA fragmentation. J Biol Chem 1996;271:21709-21712.

17 Andrade F, Roy S, Nicholson D, et al. Granzyme B directly and efficiently cleaves several downstream caspase substrates: implications for CTL-induced apoptosis. Immunity 1998;8:451-460.

18 Heibein JA, Goping IS, Barry M, et al. Granzyme Bmediated cytochrome c release is regulated by the bcl-2 family members bid and Bax. J Exp Med 2000; 192:1391-1402. 
19 Sutton VR, Davis JE, Cancilla M, et al. Initiation of apoptosis by granzyme B requires direct cleavage of bid, but not direct granzyme B-mediated caspase activation. J Exp Med 2000;192:1403-1414.

20 Alimonti JB, Shi L, Baijal PK, et al. Granzyme B induces BID-mediated cytochrome $\mathrm{C}$ release and mitochondrial permeability transition. J Biol Chem 2001;276:6974-6982.

21 International Union Against Cancer (UICC). TNM Classification of Malignant Tumors, 5th edn. UICC: Geneva, Switzerland, 1997.

22 Shanmugaratnam K (ed). Histologic Typing of Tumours of the Upper Respiratory Tract and Ear, 2nd edn. Springer Verlag: Berlin, 1991, 201pp.

23 Dukers DF, Oudejans JJ, Vos W, et al. Apoptosis in B-cell lymphomas and reactive lymphoid tissues always involves activation of caspase 3 as determined by a new in situ detection method. J Pathol 2002; 196:307-315.

24 Casciola-Rosen L, Nicholson DW, Chong T, et al. Apopain/CPP32 cleaves proteins that are essential for cellular repair: a fundamental principle of apoptotic death. J Exp Med 1996;183:1957-1964.

25 Germain M, Affar EB, D'Amours D, et al. Cleavage of automodified poly(ADP-ribose) polymerase during apoptosis. Evidence for involvement of caspase-7. J Biol Chem 1999;274:28379-28384.

26 Cox DR. Regression models and life tables. J R Stat Soc 1972;B34:187.

27 Robertson JD, Gogvadze V, Zhivotovsky B, et al. Distinct pathways for stimulation of cytochrome c release by etoposide. J Biol Chem 2000;275:3243832443.

28 Vicat JM, Ardila-Osorio H, Khabir A, et al. Apoptosis and TRAF-1 cleavage in Epstein-Barr virus-positive nasopharyngeal carcinoma cells treated with doxorubicin combined with a farnesyl-transferase inhibitor. Biochem Pharmacol 2003;65:423-433.

29 Tombolini V, De Sanctis V, Donato V, et al. Prognostic features and treatment outcome in patients with nasopharyngeal carcinoma: an experience of 20 years. Anticancer Res 2001;21:1413-1418.

30 Ma J, Mai HQ, Hong MH, et al. Is the 1997 AJCC staging system for nasopharyngeal carcinoma prognostically useful for Chinese patient populations? Int J Radiat Oncol Biol Phys 2001;50:1181-1189.

31 Lee AWM, Foo W, Law SCK, et al. Staging of nasopharyngeal carcinoma: from Ho's to the new UICC system. Int J Cancer (Pred Oncol) 1999;84: 179-187.

32 Oudejans JJ, Harijadi H, Kummer JA, et al. High numbers of granzyme B/CD8-positive tumour-infiltrating lymphocytes in nasopharyngeal carcinoma biop- sies predict rapid fatal outcome in patients treated with curative intent. J Pathol 2002;198:468-475.

33 Harn HJ, Hsieh HF, Ho LI, et al. Apoptosis in nasopharyngeal carcinoma as related to histopathological characteristics and clinical stage. Histopathology 1998;33:117-122.

34 Soengas MS, Capodieci P, Polsky D, et al. Inactivation of the apoptosis effector Apaf-1 in malignant melanoma. Nature 2001;409:207-211.

35 Los M, Herr I, Friesen C, et al. Cross-resistance of CD95- and drug-induced apoptosis as a consequence of deficient activation of caspases (ICE/Ced-3 proteases). Blood 1997;90:3118-3129.

36 Boesen-de Cock JG, Tepper AD, de Vries E, et al. Common regulation of apoptosis signaling induced by CD95 and the DNA-damaging stimuli etoposide and gamma-radiation downstream from caspase-8 activation. J Biol Chem 1999;274:14255-14261.

37 Fei P, Bernhard EJ, El-Deiry WS. Tissue-specific induction of p53 targets in vivo. Cancer Res 2002;62: 7316-7327.

38 Spruck III CH, Tsai YC, Huang DP, et al. Absence of p53 gene mutations in primary nasopharyngeal carcinomas. Cancer Res 1992;52:4787-4790.

39 Lung $\mathrm{ML}, \mathrm{Hu} \mathrm{Y}$, Cheng $\mathrm{Y}$, et al. p53 inactivating mutations in Chinese nasopharyngeal carcinomas. Cancer Lett 1998;133:89-94.

40 Ambrosini G, Adida C, Altieri DC. A novel antiapoptosis gene, survivin, expressed in cancer and lymphoma. Nat Med 1997;3:917-921.

41 Huang H, Pan X, Zhou J. BHRF1 antisense oligonucleotide inhibits anti-apoptosis of nasopharyngeal carcinoma cells. Int J Mol Med 1999;4:649-653.

42 Horner D, Lewis M, Farrell PJ. Novel hypotheses for the roles of EBNA-1 and BHRF1 in EBV-related cancers. Intervirology 1995;38:195-205.

43 Hayes DP, Brink AA, Vervoort MB, et al. Expression of Epstein-Barr virus (EBV) transcripts encoding homologues to important human proteins in diverse EBV associated diseases. Mol Pathol 1999;52:97-103.

44 Griffith TS, Anderson RD, Davidson BL, et al. Ratliff: Adenoviral-mediated transfer of the TNF-related apoptosis-inducing ligand/Apo-2 ligand gene induces tumor cell apoptosis. J Immunol 2000;165:2886-2894.

45 Mitsiades CS, Treon SP, Mitsiades N, et al. TRAIL/ Apo2 lignad selectively induces apoptosis and overcomes drug resistance in multipele myeloma: therapeutic applications. Blood 2001;98:795-804.

46 Sbih-Lammali F, Clausse B, Ardila-Osorio $\mathrm{H}$, et al. Control of apoptosis in Epstein Barr virus-positive nasopharyngeal carcinoma cells: opposite effects of CD95 and CD40 stimulation. Cancer Res 1999;59: 924-930. 